Supporting Information for

\title{
Estrogen Receptor Dependent Inhibitors of NF-kB Transcriptional Activation-1: Synthesis and Biological Evaluation of Substituted 2-Cyanopropanoic Acid Derivatives: Pathway Selective Inhibitors of NF-kB, a Potential Treatment for Rheumatoid Arthritis
}

Thomas J. Caggiano, ${ }^{\S *}$ Antony Brazzale ${ }^{\S}$ Douglas M. Ho, ${ }^{\#}$ Christina M. Kraml, ${ }^{\S}$ Eugene Trybulski, ${ }^{\pi}$ Christopher C. Chadwick, ${ }^{¥}$ Sue Chippari, ${ }^{*}$ Lisa Borges-Marcucci, ${ }^{¥}$ Amy Eckert, ${ }^{¥}$ James C.Keith, ${ }^{£}$ Thomas Kenney, ${ }^{¥}$ Douglas C. Harnish,

Chemical and Screening Sciences, Women's Health Research Institute, Carediovascular and Metabolic Disease Research, Wyeth Research, Princeton, NJ $08543^{\S}$

Department of Chemistry, Princeton University, Princeton, NJ 08544

Synthesis and Characterization of Compounds (1-21)

All NMR's were run at $400 \mathrm{MHz}$ unless indicated otherwise.

Ethyl 2-cyano-3,3-di-1-naphthylpropanoate (1) was prepared according to the method of Latif et al(11).

$\operatorname{mp} 189^{\circ} \mathrm{C}($ lit 188$)$

MS (APCI) $m / z 380(\mathrm{M}+\mathrm{H})$;

2-Cyano-3,3-di(1-naphthyl)propanoic acid (2)

Ethyl 2-cyano-3,3-di-1-naphthylpropanoate (1.5g, 4mmol) was dissolved in ethanol (100mL) and $1 \mathrm{~N} \mathrm{NaOH}(50 \mathrm{~mL})$ was added. The solution was stirred overnight. Isopropyl acetate $(100 \mathrm{~mL})$ was added followed by $2 \mathrm{~N} \mathrm{HCl}(50 \mathrm{~mL})$. The organic layer was separated and the aqueous layer was extracted with isopropyl acetate. The pooled organic layer was washed with brine, dried over $\mathrm{NaSO}_{4}$, filtered and concentrated in vacuo to yield a yellow foam. Recrystallization from ethyl acetate hexanes provided $1.3 \mathrm{~g}(3.7 \mathrm{mmol})$ of the acid as a white solid. HPLC analysis showed the material to be $96.6 \%$ pure. $\mathrm{mp} 182-184{ }^{\circ} \mathrm{C}$.

MS (ESI) $m / z 350$;

Anal. Calcd for $\mathrm{C}_{24} \mathrm{H}_{17} \mathrm{NO}_{2}$ : C, 82.03; H, 4.88; N, 3.99. Found: C, 79.79; H, 5.07; N, 3.68.

2-Cyano-3,3-di(1-naphthyl)propanamide (3)

Ethyl 2-cyano-3,3-di-1-naphthylpropanoate $(250 \mathrm{mg}, 0.66 \mathrm{mmol})$ is dissolved in THF $(2 \mathrm{~mL})$ in a Carius tube. To this was added concentrated $\mathrm{NH}_{4} \mathrm{OH}(5 \mathrm{~mL})$. The tube was stirred at room temperature for 24 hours and then was heated at $115^{\circ}$ for 18 hours. The sample was taken up in CHCl3 $(20 \mathrm{~mL})$ and was washed with saturated brine, dried over $\mathrm{NaSO}_{4}$, filtered and concentrated in vacuo. Chromatography yielded $38 \mathrm{mg}$ of the amide as a white powder.

MS (APCI) $m / z 368\left(\mathrm{M}+\mathrm{NH} 4^{+}\right)^{+}$;

Anal. Calcd for $\mathrm{C}_{24} \mathrm{H}_{18} \mathrm{~N}_{2} \mathrm{O}: \mathrm{C}, 82.26 ; \mathrm{H}, 5.18 ; \mathrm{N}, 7.99$. Found: C, 80.27; H, 5.69; N, 7.11.

Ethyl 2-cyano-2-methyl-3,3-di(1-naphthyl)propanoate (4)

A solution of 2-cyano-3,3-di-naphthalen-1-yl-propionic acid ethyl ester was dissolved in THF (3mL) and treated with sodium bis-trimethylsilyl amide $(39 \mu \mathrm{l}, 1 \mathrm{M}$ in THF) at room temperature. After 5 minutes methyl iodide was added $(9 \mu \mathrm{l})$ and the reaction was stirred for 4 hours. The reaction was quenched with $1 \mathrm{~N} \mathrm{HCl} \mathrm{(3ml)} \mathrm{and} \mathrm{was} \mathrm{diluted}$ with isopropyl acetate $(3 \mathrm{~mL})$. The organic layer was washed with bicarbonate then brine; dried over $\mathrm{Na}_{2} \mathrm{SO}_{4}$, filtered and evaporated to yield the title compound as a crystalline material. $\operatorname{mp} 186-188^{\circ} \mathrm{C}$; 
${ }^{1} \mathrm{H}$ NMR (400 MHz, DMSO-D6) $\delta 0.81(\mathrm{t}, J=7.08 \mathrm{~Hz}, 3 \mathrm{H}) 1.66(\mathrm{~s}, 3 \mathrm{H}) 4.01(\mathrm{~m}, 2 \mathrm{H}) 6.19(\mathrm{~s}, 1 \mathrm{H}) 7.32(\mathrm{td}, J=7.69$, $1.22 \mathrm{~Hz}, 1 \mathrm{H}) 7.43(\mathrm{q}, J=7.57 \mathrm{~Hz}, 2 \mathrm{H}) 7.57(\mathrm{~d}, J=7.32 \mathrm{~Hz}, 1 \mathrm{H}) 7.62(\mathrm{td}, J=7.69,3.90 \mathrm{~Hz}, 2 \mathrm{H}) 7.77$ (td, $J=7.69,0.98$ $\mathrm{Hz}, 1 \mathrm{H}) 7.89(\mathrm{~m}, 4 \mathrm{H}) 8.01(\mathrm{~d}, J=7.32 \mathrm{~Hz}, 1 \mathrm{H}) 8.10(\mathrm{~d}, J=7.08 \mathrm{~Hz}, 1 \mathrm{H}) 8.76(\mathrm{~d}, J=8.54 \mathrm{~Hz}, 1 \mathrm{H})$

MS (EI) $m / z 393$;

Anal. Calcd for $\mathrm{C}_{27} \mathrm{H}_{23} \mathrm{NO}_{2}$ : C, 82.42; H, 5.89; N, 3.56. Found: C, 82.20; H, 5.84; N, 3.52.

Anal. $\left(\mathrm{C}_{27} \mathrm{H}_{23} \mathrm{NO}_{2}\right) \mathrm{C}, \mathrm{H}, \mathrm{N}$.

\section{Ethyl 2-cyano-3-(1-naphthyl)-3-(2-nitrophenyl)propanoate (5)}

A solution of 2-cyano-3-(2-nitro-phenyl)-acrylic acid ethyl ester (492mg, $2 \mathrm{mmol})$ in THF (20mL) was treated dropwise with 1-naphthyl magnesium bromide $(9.6 \mathrm{~mL}, 2.4 \mathrm{mmol})$ at room temperature. The reaction was stirred for 3 hours, quenched with $1 \mathrm{~N} \mathrm{HCl}$ and diluted with ethyl acetate $(40 \mathrm{~mL})$. The organic layer was separated and was washed sequentially with saturated aqueous sodium bicarbonate solution and brine. The organic layer was dried over $\mathrm{Na}_{2} \mathrm{SO}_{4}$, filtered and was concentrated in vacuo. Chromatography over silica gel (20\% ethyl acetate/hexanes) yielded $221 \mathrm{mg}$ of the title compound as an viscous oil.

$$
\text { MS (APCI) } m / z 375
$$

Anal. Calcd for $\mathrm{C}_{22} \mathrm{H}_{18} \mathrm{~N}_{2} \mathrm{O}_{4}:$ C, 70.58; H, 4.85; N, 7.48. Found: C, 69.19; H, 4.87; N, 6.97.

\section{Ethyl 2-cyano-3-(2-methoxyphenyl)-3-(1-naphthyl)propanoate (6)}

The title compound was prepared according to the prodedure for ()ㅡㄴing 2-cyano-3-naphthalen-1-yl-acrylic acid ethyl ester and 2-methoxyphenyl magnesium bromide as starting materials. This provided the title compound as a low melting solid which NMR shows to be a 50:50 mixture of diastereomers

${ }^{1} \mathrm{H}$ NMR (400 MHz, DMSO-D6) $\delta 0.88(\mathrm{~m}, 3 \mathrm{H}) 3.84(\mathrm{~s}, 1.5 \mathrm{H}) 3.94(\mathrm{~s}, 1.5 \mathrm{H}) 3.99(\mathrm{~m}, 2 \mathrm{H}) 5.15(\mathrm{~d}, J=8.30 \mathrm{~Hz}, 0.5$ H) $5.25(\mathrm{~d}, J=9.27 \mathrm{~Hz}, 0.5 \mathrm{H}) 5.84(\mathrm{~d}, J=8.30 \mathrm{~Hz}, 0.5 \mathrm{H}) 5.88(\mathrm{~d}, J=9.52 \mathrm{~Hz}, 0.5 \mathrm{H}) 6.78(\mathrm{td}, J=7.44,0.98 \mathrm{~Hz}, 0.5 \mathrm{H})$ $6.88(\mathrm{td}, J=7.38,0.85 \mathrm{~Hz}, 0.5 \mathrm{H}) 6.95(\mathrm{dd}, J=7.69,1.59 \mathrm{~Hz}, 0.5 \mathrm{H}) 7.05(\mathrm{t}, J=7.69 \mathrm{~Hz}, 1 \mathrm{H}) 7.23$ (ddd, $J=15.68,7.99$, $1.71 \mathrm{~Hz}, 1 \mathrm{H}) 7.35(\mathrm{dd}, J=7.81,1.71 \mathrm{~Hz}, 0.5 \mathrm{H}) 7.52(\mathrm{~m}, 3 \mathrm{H}) 7.60(\mathrm{~d}, J=7.81 \mathrm{~Hz}, 0.5 \mathrm{H}) 7.65(\mathrm{~d}, J=7.08 \mathrm{~Hz}, 0.5 \mathrm{H})$ $7.83(\mathrm{~d}, J=8.30 \mathrm{~Hz}, 0.5 \mathrm{H}) 7.90(\mathrm{~m}, 3 \mathrm{H}) 8.11(\mathrm{~d}, J=8.79 \mathrm{~Hz}, 0.5 \mathrm{H})$

$$
\begin{aligned}
& \text { MS (EI) m/z M+. (359); } \\
& \text { Anal. }\left(\mathrm{C}_{23} \mathrm{H}_{21} \mathrm{NO}_{3}\right) \mathrm{C}, \mathrm{H}, \mathrm{N} \text {. }
\end{aligned}
$$

\section{Ethyl-(RR,SS)- 2-cyano-3-(2-methoxyphenyl)-2-methyl-3-(1-naphthyl)propanoate (7)}

A solution of 2-cyano-3-(2-methoxy-phenyl)-3-naphthalen-1-yl-propionic acid ethyl ester (205mg, 0.57mmol) in THF $(10 \mathrm{~mL})$ is cooled in a dry ice ethanol bath. A solution of KHMDS (1.04ml, $0.68 \mathrm{mmol}, 0.66 \mathrm{M}$ toluene) is added dropwise. The bath is removed and the reaction is warmed to room temperature over an hour. To this is added methyl iodide (162mg, $1.14 \mathrm{mmol}$ ) and the reaction is stirred at room temperature for 30 minutes. The reaction is quenched with $1 \mathrm{NHCl}$ and is diluted with ethyl acetate. The organic layer is isolated and is washed with saturated bicarbonate solution then brine. The organic layer is dried with $\mathrm{Na}_{2} \mathrm{SO}_{4}$, is filtered and evaporated to a solid. Chromatography over silica gel (20\%ethyl acetate/hexane) yields the title compound as a solid. Recrystallization from $\mathrm{MeOH}$ yields the title compound (80mg) as white crystals. mp 134.5-135.5 ${ }^{\circ} \mathrm{C}$;

${ }^{1} \mathrm{H}$ NMR (400 MHz, DMSO-D6) $\delta 0.75(\mathrm{t}, J=7.08 \mathrm{~Hz}, 3 \mathrm{H}) 1.65(\mathrm{~s}, 3 \mathrm{H}) 3.98(\mathrm{~m}, J=17.79,10.65,7.08,3.78 \mathrm{~Hz}, 2 \mathrm{H})$ $4.05(\mathrm{~s}, 3 \mathrm{H}) 5.81(\mathrm{~s}, 1 \mathrm{H}) 6.83(\mathrm{td}, J=7.50,1.10 \mathrm{~Hz}, 1 \mathrm{H}) 7.16(\mathrm{dt}, J=7.87,1.56 \mathrm{~Hz}, 1 \mathrm{H}) 7.26(\mathrm{td}, J=7.81,1.71 \mathrm{~Hz}, 1 \mathrm{H})$ $7.46(\mathrm{~m}, 1 \mathrm{H}) 7.58(\mathrm{dd}, J=8.18,7.44 \mathrm{~Hz}, 1 \mathrm{H}) 7.85(\mathrm{~d}, J=8.54 \mathrm{~Hz}, 1 \mathrm{H}) 7.89(\mathrm{~m}, 1 \mathrm{H}) 8.03(\mathrm{~d}, J=7.08 \mathrm{~Hz}, 1 \mathrm{H})$

$$
\begin{aligned}
& \mathrm{MS}(\mathrm{APCI}) \mathrm{m} / \mathrm{z}[\mathrm{M}+\mathrm{H}]+(374) \text {; } \\
& \text { Anal. } \mathrm{C}_{24} \mathrm{H}_{23} \mathrm{NO}_{3} \text { ) C,H N }
\end{aligned}
$$

\section{Ethyl 2-cyano-3-(1-naphthyl)-3-(2-naphthyl)propanoate (8)}

The title compound was prepared according to the prodedure for (5), using 2-cyano-3-naphthalen-1-yl-acrylic acid ethyl ester and 2-naphthyl magnesium bromide. The sample is a viscous oil. 
${ }^{1} \mathrm{H}$ NMR $(400 \mathrm{MHz}$, DMSO-D6) $\delta 0.82(\mathrm{t}, J=7.08 \mathrm{~Hz}, 1.5 \mathrm{H}) 0.89(\mathrm{t}, J=7.08 \mathrm{~Hz}, 1.5 \mathrm{H}) 3.99(\mathrm{~m}, 2 \mathrm{H}) 5.46(\mathrm{~d}, J=9.76$ $\mathrm{Hz}, 0.5 \mathrm{H}) 5.51(\mathrm{~d}, J=9.76 \mathrm{~Hz}, 0.5 \mathrm{H}) 5.70(\mathrm{~d}, J=9.76 \mathrm{~Hz}, 0.5 \mathrm{H}) 5.74(\mathrm{~d}, J=9.76 \mathrm{~Hz}, 0.5 \mathrm{H}) 7.51(\mathrm{~m}, 5 \mathrm{H}) 7.64(\mathrm{~m}, 1 \mathrm{H})$ $7.83(\mathrm{~m}, 4 \mathrm{H}) 7.93(\mathrm{~m}, 2 \mathrm{H}) 8.02(\mathrm{~d}, J=7.08 \mathrm{~Hz}, 0.5 \mathrm{H}) 8.15(\mathrm{~d}, J=1.46 \mathrm{~Hz}, 0.5 \mathrm{H}) 8.25(\mathrm{~m}, 0.5 \mathrm{H}) 8.38(\mathrm{~d}, J=8.54 \mathrm{~Hz}$, $0.5 \mathrm{H})$

$$
\text { MS (APCI) m/z } 380([\mathrm{M}+\mathrm{H}]+) \text {; }
$$

Anal. $\mathrm{C}_{26} \mathrm{H}_{21} \mathrm{NO}_{2}$ ) C, H, N

\section{Ethyl 2-cyano-3-(3-methoxyphenyl)-3-(1-naphthyl)propanoate (9)}

The title compound was prepared according to the procedure for (ㅁ), using2-cyano-3-naphthalen-1-ylacrylic acid ethyl ester and 3-methoxy-phenyl magnesium bromide. The sample is a viscous oil.

${ }^{1} \mathrm{H}$ NMR (400 MHz, DMSO-D6) $\delta 0.85(\mathrm{t}, J=7.08 \mathrm{~Hz}, 1.5 \mathrm{H}) 0.90(\mathrm{t}, J=7.20 \mathrm{~Hz}, 1.5 \mathrm{H}) 2.04(\mathrm{~s}, 1 \mathrm{H}) 3.66(\mathrm{~s}, 1.5 \mathrm{H})$ $3.67(\mathrm{~s}, 1.5 \mathrm{H}) 3.97(\mathrm{~m}, 2 \mathrm{H}) 5.30(\mathrm{~d}, J=9.52 \mathrm{~Hz}, 0.5 \mathrm{H}) 5.38(\mathrm{~d}, J=10.01 \mathrm{~Hz}, 0.5 \mathrm{H}) 5.46(\mathrm{~d}, J=9.76 \mathrm{~Hz}, 0.5 \mathrm{H}) 5.50(\mathrm{~d}$, $J=9.76 \mathrm{~Hz}, 0.5 \mathrm{H}) 6.76(\mathrm{~m}, 1 \mathrm{H}) 6.89(\mathrm{~d}, J=8.30 \mathrm{~Hz}, 0.5 \mathrm{H}) 6.97(\mathrm{t}, J=1.95 \mathrm{~Hz}, 0.5 \mathrm{H}) 7.10(\mathrm{~m}, 1 \mathrm{H}) 7.15(\mathrm{t}, J=7.93 \mathrm{~Hz}$, $0.5 \mathrm{H}) 7.20(\mathrm{t}, J=7.93 \mathrm{~Hz}, 0.5 \mathrm{H}) 7.49(\mathrm{~m}, 1.5 \mathrm{H}) 7.57(\mathrm{t}, J=7.69 \mathrm{~Hz}, 0.5 \mathrm{H}) 7.70(\mathrm{~d}, J=7.08 \mathrm{~Hz}, 0.5 \mathrm{H}) 7.81(\mathrm{~d}, J=8.05$ $\mathrm{Hz}, 0.5 \mathrm{H}) 7.90(\mathrm{~m}, 2 \mathrm{H}) 8.16(\mathrm{~d}, J=9.52 \mathrm{~Hz}, 0.5 \mathrm{H}) 8.30(\mathrm{~d}, J=8.05 \mathrm{~Hz}, 0.5 \mathrm{H})$

MS (EI) m/z M+. (359);

Anal. calcd for $\mathrm{C}_{23} \mathrm{H}_{21} \mathrm{NO}_{3}$ : C:76.86 H:5.89 N:3.90 Found: C:75.45 H:5.83 N:3.58

\section{Ethyl 2-cyano-3-(4-methylphenyl)-3-(1-naphthyl)propanoate (10)}

The title compound was prepared according to the procedure for (ㅁ) , using 2-cyano-3-naphthalen-1-yl-acrylic acid ethyl ester and 4-methylphenyl magnesium bromide. The sample is a viscous oil.

${ }^{1} \mathrm{H}$ NMR (400 MHz, DMSO-D6) $\delta 0.90(\mathrm{dt}, J=12.45,7.08 \mathrm{~Hz}, 3 \mathrm{H}) 2.06(\mathrm{~s}, 3 \mathrm{H}) 2.19(\mathrm{~s}, 1.5 \mathrm{H}) 2.22(\mathrm{~s}, 1.5 \mathrm{H}) 4.00$ (ddd, $J=13.85,7.02,4.03 \mathrm{~Hz}, 2 \mathrm{H}) 5.28(\mathrm{~d}, J=9.52 \mathrm{~Hz}, 0.5 \mathrm{H}) 5.35(\mathrm{~d}, J=9.52 \mathrm{~Hz}, 0.5 \mathrm{H}) 5.46(\mathrm{~d}, J=9.76 \mathrm{~Hz}, 0.5 \mathrm{H})$ $5.50(\mathrm{~d}, J=9.52 \mathrm{~Hz}, 0.5 \mathrm{H}) 7.09(\mathrm{dd}, J=15.13,7.81 \mathrm{~Hz}, 2 \mathrm{H}) 7.26(\mathrm{~d}, J=8.30 \mathrm{~Hz}, 1 \mathrm{H}) 7.41(\mathrm{~d}, J=8.30 \mathrm{~Hz}, 1 \mathrm{H}) 7.52(\mathrm{~m}$, $2.5 \mathrm{H}) 7.70(\mathrm{~d}, J=6.59 \mathrm{~Hz}, 0.5 \mathrm{H}) 7.83(\mathrm{~d}, J=8.05 \mathrm{~Hz}, 0.5 \mathrm{H}) 7.91(\mathrm{~m}, 1 \mathrm{H}) 8.14(\mathrm{dd}, J=5.13,4.39 \mathrm{~Hz}, 0.5 \mathrm{H}) 8.26(\mathrm{~d}$, $J=8.54 \mathrm{~Hz}, 0.5 \mathrm{H})$

MS (EI) m/z M+. (343);

Anal. calcd for $\mathrm{C}_{23} \mathrm{H}_{21} \mathrm{NO}_{2}$ : C:80.44 H:6.16 N:4.08 Found: C:78.76 H:6.32 N:3.81.

\section{Ethyl 2-cyano-3-(1-naphthyl)-3-phenylpropanoate (11)}

A solution of 2-cyano-3-naphthalen-1-yl-acrylic acid ethyl ester $(0.502 \mathrm{mg}, 2 \mathrm{mmol})$ in $\mathrm{THF}(15 \mathrm{~mL})$ was treated with phenyl magnesiun bromide $(1.2 \mathrm{~mL}, 2 \mathrm{M}$ in THF) dropwise with stirring under nitrogen. After one hour the reaction was quenched with $1 \mathrm{~N} \mathrm{HCl}$. The solution was diluted with isopropyl acetate $(15 \mathrm{~mL})$ and the organic layer was isolated. The organic layer was washed with bicarbonate solution and brine. Chromatography over silica gel (15\% MTBE/hexane) provided the title compound as an oil from which solidified on standing. NMR suggests a 6:1 ratio of diastereomers.

${ }^{1} \mathrm{H}$ NMR (400 MHz, DMSO-D6) $\delta 0.89$ (dt, $\left.J=9.70,7.11 \mathrm{~Hz}, 3 \mathrm{H}\right) 4.00$ (ddd, $J=14.28,7.08,1.10 \mathrm{~Hz}, 2 \mathrm{H}$ ) 5.33 (d, $J=9.76 \mathrm{~Hz}, 0.8 \mathrm{H}) 5.40(\mathrm{~d}, J=9.76 \mathrm{~Hz}, 0.2 \mathrm{H}) 5.51(\mathrm{~d}, J=9.76 \mathrm{~Hz}, 0.8 \mathrm{H}) 5.55(\mathrm{~d}, J=9.76 \mathrm{~Hz}, 0.2 \mathrm{H}) 7.25(\mathrm{~m}, 3.2 \mathrm{H}) 7.40$ $(\mathrm{d}, J=7.08 \mathrm{~Hz}, 1.8 \mathrm{H}) 7.52(\mathrm{~m}, 2.6 \mathrm{H}) 7.60(\mathrm{t}, J=7.81 \mathrm{~Hz}, 1 \mathrm{H}) 7.72(\mathrm{~d}, J=7.08 \mathrm{~Hz}, 0.2 \mathrm{H}) 7.84(\mathrm{~d}, J=8.79 \mathrm{~Hz}, 0.2 \mathrm{H})$ $7.90(\mathrm{~d}, J=8.05 \mathrm{~Hz}, 1 \mathrm{H}) 7.94(\mathrm{~m}, 1.8 \mathrm{H}) 8.18(\mathrm{~m}, 0.8 \mathrm{H}) 8.30(\mathrm{~d}, J=7.57 \mathrm{~Hz}, 0.2 \mathrm{H})$

MS (EI) m/z M+. (329);

Anal. $\left(\mathrm{C}_{22} \mathrm{H}_{19} \mathrm{NO}_{2}\right) \mathrm{C}, \mathrm{H}, \mathrm{N}$.

\section{Ethyl 2-cyano-3-(2-fluorophenyl)-3-(1-naphthyl)propanoate (12)}

The title compound was prepared according to the procedure for (ㅁ) , using of 2-cyano

(2-fluorophenyl)-acrylic acid ethyl ester and 1-nathphyl magnesium bromide. The

sample is a viscous oil.

MS (APCI) $m / z 348$;

Ethyl 2-cyano-3-(1-naphthyl)-3-quinolin-4-ylpropanoate (13) 
The title compound was prepared according to the procedure for (ㅁ) , using of 2-cyano-

3-quinolin-4-yl-acrylic acid ethyl ester and 1-nathphyl magnesium bromide. The

sample is a white foam.

MS (APCI) $m / z 381$;

\section{Ethyl 2-cyano-3-(1-naphthyl)-3-[2-(trifluoromethyl)phenyl]propanoate (14)}

2-(trifluoromethyl)phenyl magnesium bromide (550 mg, $2 \mathrm{mmol}$ ) taken up in $10 \mathrm{~mL}$ dry THF with magnesium (59 $\mathrm{mg}, 2 \mathrm{mmol}$ ) overnight. To this was added ethyl (E)-2-cyano-3-(1-naphthyl)prop-2-enoate (502 mg, $2 \mathrm{mmol}$ ) in THF $(10 \mathrm{~mL})$. The reaction mixture allowed to stir overnight. The reaction was stirred $2 \mathrm{hrs}$, quenched with $1 \mathrm{~N} \mathrm{HCl}$, taken up in ethyl acetate, washed with $\mathrm{NaHCO}_{3}$, brine, dried with $\mathrm{MgSO}_{4}$, filtered, and evaporated. The crude reaction mixture was purified on silica gel (20\% ethyl acetate/hexanes) to yield $692 \mathrm{mg}$ of the title compound as a yellow oil.

${ }^{1} \mathrm{H}$ NMR (400 MHz, DMSO-D6) $\delta 0.76(\mathrm{t}, J=7.08 \mathrm{~Hz}, 1 \mathrm{H}) 0.92(\mathrm{t}, J=7.08 \mathrm{~Hz}, 1 \mathrm{H}) 3.87(\mathrm{~m}, 1 \mathrm{H}) 4.02(\mathrm{q}, J=7.08$ $\mathrm{Hz}, 0.5 \mathrm{H}) 4.07(\mathrm{q}, J=7.08 \mathrm{~Hz}, 0.5 \mathrm{H}) 5.23(\mathrm{~d}, J=7.32 \mathrm{~Hz}, 0.5 \mathrm{H}) 5.36(\mathrm{~d}, J=8.30 \mathrm{~Hz}, 0.5 \mathrm{H}) 5.87(\mathrm{~d}, J=7.32 \mathrm{~Hz}, 0.5 \mathrm{H})$ $5.94(\mathrm{~d}, J=8.30 \mathrm{~Hz}, 0.5 \mathrm{H}) 7.27(\mathrm{~d}, J=6.59 \mathrm{~Hz}, 0.5 \mathrm{H}) 7.59(\mathrm{~m}, 5 \mathrm{H}) 7.80(\mathrm{~m}, 1.5 \mathrm{H}) 7.94(\mathrm{~m}, 3 \mathrm{H}) 8.13(\mathrm{~d}, J=8.79 \mathrm{~Hz}$, $0.5 \mathrm{H}) 8.18(\mathrm{~d}, J=7.81 \mathrm{~Hz}, 0.5 \mathrm{H})$

MS (APCI) m/z $398([\mathrm{M}+\mathrm{H}]+)$;

Anal. calcd for $\mathrm{C}_{23} \mathrm{H}_{18} \mathrm{~F}_{3} \mathrm{NO}_{2}$ : C:69.52 H:4.57 N:3.52 Found: C:68.50 H:4.09 N:3.22.

\section{Ethyl 2-cyano-3-(2,4-dimethoxyphenyl)-3-(1-naphthyl)propanoate (15)}

A solution of 2-cyano-3-(2,4-dimethoxy-phenyl)-acrylic acid ethyl ester (522mg, 2mmol) in THF (20mL) was treated dropwise with 1-naphthyl magnesium bromide $(9.6 \mathrm{~mL}, 2.4 \mathrm{mmol})$ at room temperature. The reaction was stirred for 3 hours, quenched with $1 \mathrm{~N} \mathrm{HCl}$ and diluted with ethyl acetate $(40 \mathrm{~mL})$. The organic layer was separated and was washed sequentially with saturated aqueous sodium bicarbonate solution and brine. The organic layer was dried over $\mathrm{Na}_{2} \mathrm{SO}_{4}$, filtered and was concentrated in vacuo. Chromatography over silica gel (20\% ethyl acetate/hexanes) yielded $421 \mathrm{mg}$ of the title compound as an off white solid.

${ }^{1} \mathrm{H}$ NMR (400 MHz, DMSO-D6) $\delta 0.90(\mathrm{td}, J=7.08,1.71 \mathrm{~Hz}, 3 \mathrm{H}) 3.68(\mathrm{~s}, 1.5 \mathrm{H}) 3.71(\mathrm{~s}, 1.5 \mathrm{H}) 3.84(\mathrm{~s}, 1.5 \mathrm{H}) 3.94$ $(\mathrm{s}, 1.5 \mathrm{H}) 4.00(\mathrm{~m}, 2 \mathrm{H}) 5.08(\mathrm{~d}, J=8.30 \mathrm{~Hz}, 0.5 \mathrm{H}) 5.20(\mathrm{~d}, J=9.27 \mathrm{~Hz}, 0.5 \mathrm{H}) 5.74(\mathrm{~d}, J=8.54 \mathrm{~Hz}, 0.5 \mathrm{H}) 5.78(\mathrm{~d}, J=9.27$ $\mathrm{Hz}, 0.5 \mathrm{H}) 6.36(\mathrm{dd}, J=8.54,2.44 \mathrm{~Hz}, 0.5 \mathrm{H}) 6.45(\mathrm{dd}, J=8.66,2.32 \mathrm{~Hz}, 0.5 \mathrm{H}) 6.60(\mathrm{dd}, J=8.18,2.32 \mathrm{~Hz}, 1 \mathrm{H}) 6.80(\mathrm{~d}$, $J=8.54 \mathrm{~Hz}, 0.5 \mathrm{H}) 7.20(\mathrm{~d}, J=8.54 \mathrm{~Hz}, 0.5 \mathrm{H}) 7.51(\mathrm{~m}, 3.5 \mathrm{H}) 7.64(\mathrm{~d}, J=6.83 \mathrm{~Hz}, 0.5 \mathrm{H}) 7.88(\mathrm{~m}, 3.5 \mathrm{H}) 8.06(\mathrm{~d}, J=8.54$ $\mathrm{Hz}, 0.5 \mathrm{H})$

MS (APCI) m/z $390([\mathrm{M}+\mathrm{H}]+)$;

Anal. $\left(\mathrm{C}_{24} \mathrm{H}_{23} \mathrm{NO}_{4}\right) \mathrm{C}, \mathrm{H}, \mathrm{N}$.

Ethyl 2-cyano-3-[2-(methylthio)phenyl]-3-(1-naphthyl)propanoate (16)

The title compound was prepared according to the procedure for (14) using 2-(thiomethyl)phenyl magnesium bromide. The sample is a sticky white foam

${ }^{1} \mathrm{H}$ NMR (400 MHz, DMSO-D6) $\delta 0.91(\mathrm{dt}, J=16.23,7.14 \mathrm{~Hz}, 3 \mathrm{H}) 4.01(\mathrm{~m}, 2 \mathrm{H}) 5.36(\mathrm{~d}, J=9.52 \mathrm{~Hz}, 0.5 \mathrm{H}) 5.43(\mathrm{~d}$, $J=9.76 \mathrm{~Hz}, 0.5 \mathrm{H}) 5.56(\mathrm{~d}, J=9.52 \mathrm{~Hz}, 0.5 \mathrm{H}) 5.60(\mathrm{~d}, J=10.01 \mathrm{~Hz}, 0.5 \mathrm{H}) 7.39(\mathrm{~m}, 3 \mathrm{H}) 7.55(\mathrm{~m}, 4 \mathrm{H}) 7.72(\mathrm{~d}, J=6.83$ $\mathrm{Hz}, 0.5 \mathrm{H}) 7.85(\mathrm{~d}, J=8.30 \mathrm{~Hz}, 0.5 \mathrm{H}) 7.92(\mathrm{~m}, 2 \mathrm{H}) 8.17(\mathrm{~m}, 0.5 \mathrm{H}) 8.29(\mathrm{~d}, J=8.54 \mathrm{~Hz}, 0.5 \mathrm{H})$

MS (APCI) m/z [M+H]+ (376);

Anal. $\left(\mathrm{C}_{23} \mathrm{H}_{21} \mathrm{NO}_{2} \mathrm{~S}\right) \mathrm{C}, \mathrm{H}, \mathrm{S}, \mathrm{N}$.

\section{Ethyl (RR, SS)-2-cyano-2-methyl-3-[2-(methylthio)phenyl]-3-(1-naphthyl)propanoate (17)}

The title compound was prepared according to the procedure for (7) using ethyl 2-cyano-3-[2-(methylthio)phenyl]3 -(1-naphthyl)propanoate The title compound is obtained as white crystals from $\mathrm{MeOH}$.

$\operatorname{mp} 154-156^{\circ} \mathrm{C}$; 
MS (APCI) $m / z 390$;

nal. $\left(\mathrm{C}_{24} \mathrm{H}_{23} \mathrm{NO}_{2} \mathrm{~S}\right) \mathrm{C}, \mathrm{H}, \mathrm{S}, \mathrm{N}$.

\section{tert-Butyl (RR,SS)-2-cyano-3-(2-methoxyphenyl)-2-methyl-3-(1-naphthyl)propanoate (18)}

A solution of 2-cyano-3-(2-methoxy-phenyl)-3-naphthalen-1-yl-propionic acid tert-butyl ester in THF ( $25 \mathrm{~mL}$ ) was treated at room temperature with potassium bistrimethylsilyl amide $(3.64 \mathrm{~mL}, 2.4 \mathrm{mmol}, 0.66 \mathrm{M}$ in toluene). The reaction was stirred for two hours and then methyl iodide was added $(0.5 \mathrm{~mL}, 8 \mathrm{mmol})$. The reaction was stirred at room temperature for one hour and was quenched with $1 \mathrm{~N} \mathrm{HCl}$. The reaction was diluted with ethyl acetate and the organic layer was isolated. The organic layer was washes with saturated sodium bicarbonate solution and brine. The organic layer was dried over $\mathrm{Na}_{2} \mathrm{SO}_{4}$, filtered and was concentrated in vacuo. Trituration of the resulting solid with ethanol provided $700 \mathrm{mg}$ of the title compound as a white powder.

${ }^{1} \mathrm{H}$ NMR (400 MHz, DMSO-D6) $\delta 1.02(\mathrm{~s}, 9 \mathrm{H}) 1.62(\mathrm{~s}, 3 \mathrm{H}) 4.04(\mathrm{~s}, 3 \mathrm{H}) 5.76(\mathrm{~s}, 1 \mathrm{H}) 6.83(\mathrm{td}, J=7.50,1.10 \mathrm{~Hz}, 1$ H) $7.21(\mathrm{~m}, 3 \mathrm{H}) 7.47(\mathrm{~m}, 2 \mathrm{H}) 7.60(\mathrm{t}, J=7.32 \mathrm{~Hz}, 1 \mathrm{H}) 7.89(\mathrm{~m}, 3 \mathrm{H}) 8.06(\mathrm{~d}, J=7.08 \mathrm{~Hz}, 1 \mathrm{H})$

MS (APCI) m/z $402([\mathrm{M}+\mathrm{H}]+)$;

Anal. calcd for $\mathrm{C}_{26} \mathrm{H}_{27} \mathrm{NO}_{3}$ : C:77.78 H:6.78 N:3.49 Found: C:77.41 H:6.84 N:3.67.

Tert-Butyl (SS)-2-cyano-3-(2-methoxyphenyl)-2-methyl-3-(1-naphthyl)propanoate

tert-Butyl (RR)-2-cyano-3-(2-methoxyphenyl)-2-methyl-3-(1-naphthyl)propanoate

The ester was resolved into its enantiomers by preparative chiral chromatography on a Chiralcel OD column in 80/20 methanol /water. The first peak to elute is (+) tert-butyl (SS)-2-cyano-3-(2-methoxyphenyl)-2-methyl-3-(1naphthyl)propanoate

Chemical purity $=99.9 \%$ with ee $100 \%$.

$[\alpha]_{\mathrm{D}}^{25}=-283.8^{\circ}\left(\mathrm{c}=5.624 \mathrm{mg} / 1.124 \mathrm{~mL}, \mathrm{CHCl}_{3}\right)$;

The second peak to elute is (-)tert-butyl (SS)-2-cyano-3-(2-methoxyphenyl)-2-methyl-3-(1-naphthyl)propanoate

Chemical purity $=98 \%$ with ee $96 \%$

$[\alpha]_{\mathrm{D}}^{25}=+272.0^{\circ}\left(\mathrm{c}=5.399 \mathrm{mg} / 1.080 \mathrm{~mL}, \mathrm{CHCl}_{3}\right) ;$

(RR,SS)-3-(2-Methoxyphenyl)-2-methyl-3-(1-naphthyl)-2-(piperidin-1-ylcarbonyl)propanenitrile (19)

(RR,SS)-2-cyano-3-(2-methoxyphenyl)-2-methyl-3-(1-naphthyl)propanoic acid(1 g, $2.89 \mathrm{mmol}$ ) was dissolved in 50 $\mathrm{mL}$ THF. A catalytic amount of DMF was added to the reaction mixture. Oxalyl chloride $(0.303 \mathrm{~mL}, 3.47 \mathrm{mmol})$ was added slowly. The reaction mixture was stirred until no more evolution of gas was observed. The reaction mixture was rapidly heated to reflux, then cooled and evaporated. $50 \mathrm{~mL}$ toluene was added and the reaction mixture was evaporated to dryness. The acid chloride was dissolved in $10 \mathrm{~mL} \mathrm{CH}_{2} \mathrm{Cl}_{2}$ and placed under nitrogen. In a separate flask piperidine $(0.49 \mathrm{~mL}, 4.91 \mathrm{mmol})$ was dissolved in $50 \mathrm{~mL} \mathrm{CH}_{2} \mathrm{Cl}_{2}$ under nitrogen. To this stirred solution was added a catalytic amount of DMAP, and triethylamine $(0.69 \mathrm{~mL}, 4.91 \mathrm{mmol})$. The acid chloride in $\mathrm{CH}_{2} \mathrm{Cl}_{2}$ was added rapidly to this stirred mixture and allowed to react for 2 hours after which water was added to the reaction mixture. The organic layer was separated and washed with brine, dried over $\mathrm{MgSO}_{4}$, filtered, evaporated to dryness, and recrystallized from ethanol to yield $851 \mathrm{mg}$ of the title compound as a crystalline solid.

$\operatorname{mp} 194-196{ }^{\circ} \mathrm{C}$;

${ }^{1} \mathrm{H}$ NMR $400 \mathrm{MHz}$ (DMSO-D6): $\delta 7.94$ (d, 1H, J=7.32 Hz), 7.85 (m, 2H), 7.78 (d, 1H, J=8.30 Hz), 7.52 (t, $1 \mathrm{H}, \mathrm{J}=7.32$ $\mathrm{Hz}), 7.40$ (m, 2H), $7.21(\mathrm{td}, 1 \mathrm{H}, \mathrm{J}=7.75 \mathrm{~Hz}, 1.71 \mathrm{~Hz}), 7.12(\mathrm{dd}, 1 \mathrm{H} \mathrm{J}=8.30 \mathrm{~Hz}, 0.98 \mathrm{~Hz}), 7.06$ (dd, 1H, J=7.81 Hz, 1.71 $\mathrm{Hz}), 6.77(\mathrm{td}, 1 \mathrm{H}, \mathrm{J}=7.57 \mathrm{~Hz}, 0.98 \mathrm{~Hz}), 5.99(\mathrm{~s}, 1 \mathrm{H}) 4.01(\mathrm{~s}, 3 \mathrm{H}), 3.49(\mathrm{bs}, 4 \mathrm{H}), 1.58(\mathrm{~s}, 1 \mathrm{H}), 1.41(\mathrm{bs}, 6 \mathrm{H})$

MS (APCI) m/z $413([\mathrm{M}+\mathrm{H}]+)$;

(2S)-3-[4-(3-Chloro-2-methylphenyl)piperazin-1-yl]-2-[(S)-(2-methoxyphenyl)(1-naphthyl)methyl]-2methyl-3-oxopropanenitrile (20) 
(S,S)-2-cyano-3-(2-methoxyphenyl)-2-methyl-3-(1-naphthyl)propanoic acid(12 g, $34.74 \mathrm{mmol}$ ) was dissolved in 500 $\mathrm{mL}$ THF. A catalytic amount of DMF was added to the reaction mixture. Oxalyl chloride (3.64 $\mathrm{mL}, 41.69 \mathrm{mmol})$ was added slowly. The reaction mixture was stirred until no more evolution of gas was observed. The reaction mixture was rapidly heated to reflux, then cooled and evaporated. $250 \mathrm{~mL}$ toluene was added and the reaction mixture was evaporated to dryness. The acid chloride was dissolved in $250 \mathrm{~mL} \mathrm{CH}_{2} \mathrm{Cl}_{2}$ and placed under nitrogen. In a separate flask 1-(3-chloro-2-methylphenyl)piperazine hydrochloride (10.67 g, $41.69 \mathrm{mmol}$ ) was dissolved in 250 $\mathrm{mL} \mathrm{CH}_{2} \mathrm{Cl}_{2}$ under nitrogen. To this stirred solution was added a catalytic amount of DMAP, and triethylamine $(21.80 \mathrm{~mL}, 156.33 \mathrm{mmol})$. The acid chloride in $\mathrm{CH}_{2} \mathrm{Cl}_{2}$ was added rapidly to this stirred mixture and allowed to react overnight after which water was added to the reaction mixture. The organic layer was separated and washed with brine, dried over $\mathrm{MgSO}_{4}$, filtered, evaporated to dryness, purified on silica gel ( $20 \%$ hexanes $/ \mathrm{CH}_{2} \mathrm{Cl}_{2}$ ), the purified fractions were evaporated and recrystallized from ethyl acetate/hexanes to yield $12.58 \mathrm{~g}$ of the title compound as a crystalline solid.

$\operatorname{mp} 150-153{ }^{\circ} \mathrm{C}$;

$[\alpha]_{\mathrm{D}}^{25}=-167.9^{\circ}\left(1 \%, \mathrm{CHCl}_{3}\right)$;

${ }^{1} \mathrm{H}$ NMR $500 \mathrm{MHz}$ (DMSO-D6): • 8.00 (d, 1H, J=7.33 Hz), $7.88(\mathrm{~m}, 2 \mathrm{H}), 7.86(\mathrm{~d}(1 \mathrm{H}, \mathrm{J}=8.25 \mathrm{~Hz}), 7.55$ (t, $1 \mathrm{H}, \mathrm{J}=7.64$ $\mathrm{Hz}), 7.43(\mathrm{~m}, 2 \mathrm{H}), 7.23(\mathrm{td}, 1 \mathrm{H}, \mathrm{J}=7.79 \mathrm{~Hz}, 1.37 \mathrm{~Hz}), 7.14(\mathrm{~m}, 3 \mathrm{H}), 6.86(\mathrm{bs}, 1 \mathrm{H}), 6.80(\mathrm{t}, 1 \mathrm{H}, \mathrm{J}=7.48 \mathrm{~Hz}), 6.02(\mathrm{~s}, 1 \mathrm{H})$, $4.01(\mathrm{~s}, 3 \mathrm{H}), 3.69(\mathrm{bs}, 4 \mathrm{H}), 2.70(\mathrm{bs}, 4 \mathrm{H}), 2.27(\mathrm{~s}, 3 \mathrm{H}), 1.65(\mathrm{~s}, 3 \mathrm{H})$

MS (ESI) m/z $538([\mathrm{M}+\mathrm{H}]+)$;

Anal. $\left(\mathrm{C}_{33} \mathrm{H}_{32} \mathrm{ClN}_{3} \mathrm{O}_{2}\right) \mathrm{C}, \mathrm{H}, \mathrm{N}$.

(2S,3S)-3-(2-methoxyphenyl)-2-methyl-3-(1-naphthyl)-2-(\{4-[3-(trifluoromethyl)phenyl]piperidin-1yl\} carbonyl)propanenitrile (21)

The title compound was prepared in $75 \%$ yield according to the procedure for $\underline{\mathbf{( 2 0})}$ using 4 -(3-trifluoromethylphenyl)-piperidine. Recrystallization from ethyl acetate/hexane yielded white crystals $\operatorname{mp} 123-127^{\circ} \mathrm{C}$;

$[\alpha]_{\mathrm{D}}^{25}=-144.4^{\circ}\left(1 \%, \mathrm{CHCl}_{3}\right)$;

${ }^{1} \mathrm{H}$ NMR 500MHz (DMSO-D6): • $8.02(\mathrm{~d}, 1 \mathrm{H}, \mathrm{J}=7.03 \mathrm{~Hz}), 7.88(\mathrm{~m}, 2 \mathrm{H}), 7.82(\mathrm{~d}, 1 \mathrm{H}, \mathrm{J}=8.25 \mathrm{~Hz}), 7.56(\mathrm{t}, 1 \mathrm{H}, \mathrm{J}=$ $7.79 \mathrm{~Hz}), 7.51(\mathrm{brm}, 2 \mathrm{H}), 7.42(\mathrm{~d}, 1 \mathrm{H}, \mathrm{J}=7.48 \mathrm{~Hz}), 7.42($ quin, $1 \mathrm{H}, \mathrm{J}=3.51 \mathrm{~Hz}), 7.23(\mathrm{t}, 1 \mathrm{H}, \mathrm{J}=7.03 \mathrm{~Hz}), 7.14(\mathrm{~m}, 2 \mathrm{H}), 6.80$ $(\mathrm{t}, 1 \mathrm{H}, \mathrm{J}=7.48 \mathrm{~Hz}), 6.02(\mathrm{~s}, 1 \mathrm{H}), 4.37(\mathrm{brm}, 2 \mathrm{H}), 4.01(\mathrm{~s}, 3 \mathrm{H}), 2.88(\mathrm{brm}, 2 \mathrm{H}), 1.66(\mathrm{~s}, 3 \mathrm{H})$ MS (ESI) $m / z 557([\mathrm{M}+\mathrm{H}]+)$;

Anal. calcd for $\mathrm{C}_{34} \mathrm{H}_{31} \mathrm{~F}_{3} \mathrm{~N}_{2} \mathrm{O}_{2}$ : C:73.35 H:5.61 N:5.03 Found: C:73.99 H:6.00 N:4.72

(RR,SS)-2-cyano-3-(2-methoxyphenyl)-2-methyl-3-(1-naphthyl)propanoic acid

Ethyl-(RR,SS)- 2-cyano-3-(2-methoxyphenyl)-2-methyl-3-(1-naphthyl)propanoate (12.42g, $33.33 \mathrm{mmol}$ ) was dissolved in $100 \mathrm{~mL}$ THF to which was added $2.5 \mathrm{M} \mathrm{NaOH}(18 \mathrm{~mL})$. Reaction mixture heated to reflux for $8 \mathrm{hrs}$, allowed to cool and stir overnight. The reaction mixture was acidified with $1 \mathrm{~N} \mathrm{HCl}$, taken up in ethyl acetate, washed with brine, dried with $\mathrm{MgSO}_{4}$, filtered, evaporated, and recrystallized from ethanol to yield $11.44 \mathrm{~g}$ the title compound as a crystalline solid.

$\mathrm{mp} 229^{\circ} \mathrm{C}(\mathrm{dec})$;

1H NMR (400 MHz, DMSO-D6) • $1.62(\mathrm{~s}, 2 \mathrm{H}) 4.05(\mathrm{~s}, 2 \mathrm{H}) 5.77(\mathrm{~s}, 1 \mathrm{H}) 6.80(\mathrm{td}, J=7.50,1.10 \mathrm{~Hz}, 1 \mathrm{H}) 7.11(\mathrm{dd}$, $J=7.69,1.59 \mathrm{~Hz}, 1 \mathrm{H}) 7.15(\mathrm{dd}, J=8.30,0.98 \mathrm{~Hz}, 1 \mathrm{H}) 7.23(\mathrm{td}, J=7.81,1.71 \mathrm{~Hz}, 1 \mathrm{H}) 7.44(\mathrm{~m}, 1 \mathrm{H}) 7.59(\mathrm{~m}, 1 \mathrm{H}) 7.84$ $(\mathrm{d}, J=8.30 \mathrm{~Hz}, 1 \mathrm{H}) 7.89(\mathrm{~m}, 1 \mathrm{H}) 8.10(\mathrm{~d}, J=7.32 \mathrm{~Hz}, 1 \mathrm{H})$

MS (APCI) m/z 344 ([M-H]);

Anal. $\left(\mathrm{C}_{22} \mathrm{H}_{19} \mathrm{NO}_{3}\right) \mathrm{C}, \mathrm{H}, \mathrm{N}$

Resolution of (-)-(2S, 3S)-2-Cyano-3-(2-methoxyphenyl)-2-methyl-3-(1-naphthyl)propanoic Acid

\section{(2S, 3S)-2-Cyano-3-(2-methoxyphenyl)-2-methyl-3-(1-naphthyl)propanoic Acid Quinidine Salt}

2-Cyano-3-(2-methoxyphenyl)-2-methyl-3-(1-naphthyl) propanoic acid (30 g, 87 mmoles ) was dissolved in THF (250mL). This was added to a solution of quinidine $(28.18 \mathrm{~g}, 87 \mathrm{mmol})$ in $\mathrm{CH}_{2} \mathrm{Cl}_{2}(150 \mathrm{~mL})$ and isopropyl acetate $(200$ $\mathrm{mL}$ ). The solution was warmed to reflux to yield a clear solution, was cooled and evaporated to a solid in vacuo. The salt was refluxed in isopropyl acetate $(1.4 \mathrm{~L})$, filtered hot and cooled to room temperature. This provided $19.50 \mathrm{~g}$ of the diasteriomeric salt with an ee of $98.5 \%$. A second recrystallization from isopropyl acetate provided $17.5 \mathrm{~g}$ of the salt with an ee $99.5 \%$ 
mpt $171-172$

$[\alpha]_{\mathrm{D}}^{25}=-11.26^{\circ}\left(\mathrm{c}=1 \%\right.$ SOLUTION, $\left.\mathrm{CHCl}_{3}\right)$;

MS (ESI) m/z 344;

Anal. $\left(\mathrm{C}_{42} \mathrm{H}_{43} \mathrm{~N}_{3} \mathrm{O}_{5}\right) \mathrm{C}, \mathrm{H}, \mathrm{N}$.

\section{(-)-(2S, 3S)-2-Cyano-3-(2-methoxyphenyl)-2-methyl-3-(1-naphthyl)propanoic Acid}

The quinidine salt prepared in Part One was liberated to the acid by extraction with ethyl acetate / $1 \mathrm{~N}$ hydrochloric acid. The organic phase was washed with brine, dried over magnesium sulfate and filtered. The filtrate was concentrated in vacuo to a foam which was taken up in hot ether and treated with an equal volume of hexane ( $100 \mathrm{~mL}$ ) The sample were cooled in a refrigerator for 15 hours. Filtration afforded the title compound as a white crystalline solid. $5.46 \mathrm{~g}, 99.9 \%$ ee, chemical purity:99.2\%)

mp $230-233{ }^{\circ} \mathrm{C}$ dec;

$[\alpha]_{\mathrm{D}}^{25}=-270.0^{\circ}\left(\mathrm{c}=1 \%\right.$ SOLUTION, $\left.\mathrm{CHCl}_{3}\right) ;$

MS (ESI) m/z 344;

Anal. $\left(\mathrm{C}_{22} \mathrm{H}_{19} \mathrm{NO}_{3}\right) \mathrm{C}, \mathrm{H}, \mathrm{N}$ 